\title{
Téoros
}

Revue de recherche en tourisme

\section{L'industrie touristique autochtone en plein essor}

\section{Michel Noël et Lucie K. Morisset}

Volume 17, numéro 2, été 1998

L'industrie touristique autochtone

URI : https://id.erudit.org/iderudit/1072266ar

DOI : https://doi.org/10.7202/1072266ar

Aller au sommaire du numéro

Éditeur(s)

Université du Québec à Montréal

ISSN

0712-8657 (imprimé)

1923-2705 (numérique)

Découvrir la revue

Citer ce document

Noël, M. \& Morisset, L. K. (1998). L'industrie touristique autochtone en plein essor. Téoros, 17(2), 3-4. https://doi.org/10.7202/1072266ar d'utilisation que vous pouvez consulter en ligne.

https://apropos.erudit.org/fr/usagers/politique-dutilisation/ 


\section{$+\ldots$ \\ L'INDUSTRIE TOURISTIQUE AUTOCHTONE EN PLEIN ESSOR}

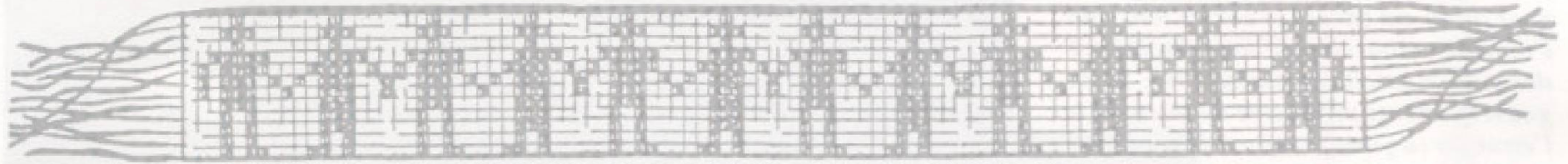

Wampum de l'alliance entre les peuples : symbole de la nécessaire collaboration entre les partenaires de l'industrie?

Michel Noël, rédacteur invité

\section{et Lucie K. Morisset}

Tout un chacun s'empresse de dire et d'écrire que l'industrie touristique des peuples autochtones du Québec est jeune, inexpérimenté ; qu'il lui revient encore de faire ses preuves. Certes vraie, cette affirmation doit être nuancée.

Dans l'univers du tourisme, les peuples amérindiens sont à la fois jeunes et anciens: anciens, car personne ne peut contester l'âge de leur présence, plusieurs fois millénaire, sur un continent qu'ils connaissent à l'évidence mieux que qui= conque; jeunes, car ils ont fait, au cours du présent siècle et particulièrement depuis 1950, un saut considérable pour tomber de plein pied dans ce qu'il est convenu d'appeler * la modernité *.

\section{Une expérience pertinente}

Les Amérindiens et les Inuit ont beaucoup plus d'experience dans le domaine touristique qu'on ne le croit généralement. Certains amérindiens pince-sans-rire (ils sont nombreux) vont jusqu'à identifier leurs premiers touristes en Jacques Cartier et son équipage : ceux-ci auraient ête tant charmés par l'accueil de l'Amérique que leurs descendants y séjourneraient en= core 500 ans plus tard!

Dans un horizon contemporain, il apparait que l'expérience des Amérindiens et des Inuit provienne des * clubs privés * : les hommes servaient de guides pour la péche ou la chasse et les femmes travaillaient aux cuisines ou au ménage des camps. Toutes les monographies des grandes " rivières à saumon : du Québec évoquent d'ailleurs les fameux guides innu (Côte Nord) et micmacs (Gaspésie). L'histoire parle aussi des Hurons (chasse à l'orignal) dont certains, devenus aussi célèbres que les rivières ou les territoires où ils ont auvré, appartiennent dorénavant aux légendes qui forgent le patrimoine collectif du Québec contemporain.

\section{La prise en charge}

Depuis une vingtaine d'années, l'association historique entre les autochtones et le tourisme a pris une nouvelle forme, radicalement différente. Tout en conservant les postes de guides qui faisaient leur réputation, les autochtones sont devenus propriétaires et promoteurs de l'industric touristique. Cette prise en charge s'est d'abord manifestée dans le domaine des pourvoiries dont plusieurs ont été créées par les autochtones ou sont passées entre leurs mains.

Puis, rapidement, le tourisme autochtone a pris une orientation davantage culturelle, voire ethnoculturelle. L'industrie touristique autochtone s'est ainsi rapidement adaptée à une nouvelle tendance touristique provenant particulièrement des pays européens, la France et l'Allemagne en tête. Aucunement fortuite, cente opportunité n'est pas non plus le fruit d'un simple calcul : les nouveaux voyages à la " Marco Polo * correspondent en effet davantage à la conception autochtone du tourisme, selon laquelle le touriste est d'abord un a visiteur s venu s'intégrer à une société étrangère via une expérience de vie familiale. Profitable pour l'industrie, cette tendance en émergence comporte surtout l'avantage de se révéler un véritable outil de * développement durable $\$$ de la culture : plus que jamais, elle donne la parole aux autochtones tout en leur permettant de recycler leurs territoires (réels et imaginaires) et leur donner une nouvelle vocation : servir le tourisme culturel. En outre, un tel tourisme soulage le cheptel de la pression exercée par la demande en matière de chasse et de pêche, devenant ainsi un atout écologique.

\section{Une conception originale}

Les autochtones entretiennent certes une conception originale du tourisme; celleci apparait d'ailleurs comme ayant con tribue de façon déterminante aux réflexions qui ont cours actuellement dans les différents ministères et les universités au regard des nouvelles tendances du tourisme. Par-delà l'industrie, les autochtones ont une approche globale de cette activité : elle ne leur semble ni sectorielle,
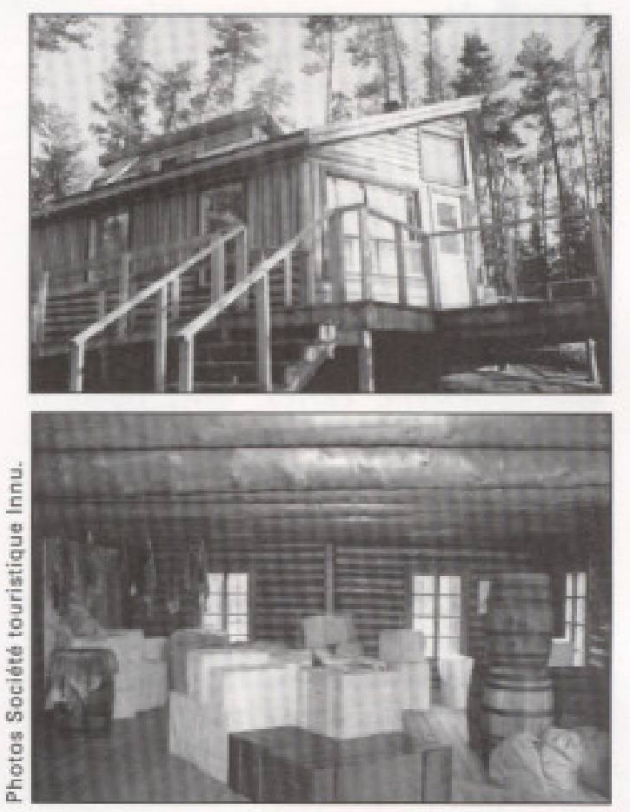

Camp moderne en billes de bois. 
ni simplement partie de la seule vie économique, mais plutôt iniégrée notamment à la vie culturelle, sociale, politique, d'où, on le comprend, l'inexorable lien qui se tisse chaque jour entre l'industrie touristique autochtone et le developpement intégré, globalisant, de la communauté.

\section{Envers et contre tous}

Cela etant dit, il est important de souligner que c'est envers et contre tous que l'industrie touristique autochtone est devenue ce qu'elle est aujourd'hui. Si les gouvernements proposent maintenant à l'étranger (à grands renforts de publicité) un tout nouveau produit : * vivre la fascinante aventure indienne en Amérique s. ce n'est pas par clairvoyance ni même par perspicacité. Comme c'est si souvent le cas, la pression est venue de l'extérieur. de la clientèle européenne qui, réclamant un tel type de tourisme, a forcé l'appareil d'État à s'adapter pour finalement inscrire les * lieux autochtones $*$ au catalogue des destinations possibles. Les décideurs et les promoteurs de l'industrie touristique au Quebec - qui jusque-là, tradition etatique oblige, se cantonnaient spontanément dans les limites du paradigme québecois - ont été littéralement renversés par le raz-de-maré que l'ignorance de l'histoire, de la culture, des modes et des milieux de vie des peuples autochtones les empêchait de voir venir.

On ne s'en cachera pas : l'industrie touristique autochtone est encore victime des préjugés qui prétendent que ses hôtesles aulochtones - ne soient pas intéressés à recevoir des touristes, qu'il y ait chez eux des problèmes d'accueil, d'hygiène, de qualité du produit ; qu'une véritable gastronomie soit absente, etc. Mais aujourd'hui, si nous souhaitons collectivement nager sur la vague, c'est en aval du produit que l'essor de l'industrie touristique autochtone paraî se jouer, sur deux volets : le soutien économique au développement, certes, mais aussi le soutien logistique à la formation et à l'organisation du marché.

\section{Du potentiel à l'essor}

L'industrie touristique autochtone ne fait en effet que commencer à recevoir une aide gouvernementale à l'echelle de son potentiel et cetle aide est loin de suffire aux besoins. Tant au fédéral qu'au provincial, les programmes d'aide sont rares et la porte qui y donne accès est étroite.

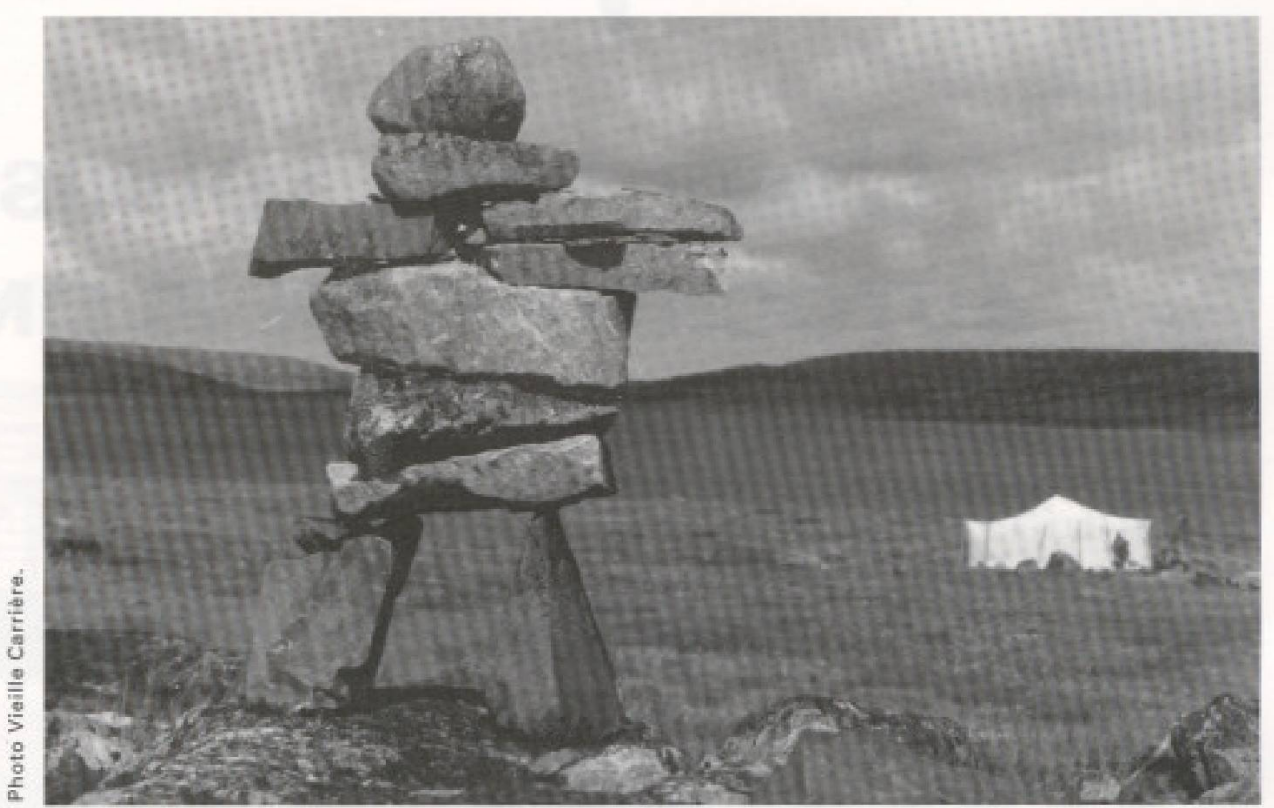

Inukshuk, puvirnifuk.

Enfin, il y a peu de spécialistes pour conseiller l'industrie naissante en matière de formation des ressources humaines ainsi qu'en ce qui concerne la mise en marché du produit. Ainsi, tous les promoteurs s'interrogent sur les façons de faire connaitre leur produit auprès de la clientèle. La question est d'autant plus pertinente que les entreprises concernées sont souvent eloignées, voire mẻme isolées, el que les coûts du marketing sont toujours exorbitants. Ce motif seul suffirait à susciter l'espoir que les gouvernements mettent rapidement en place des mesures appropriées pour appuyer le développement d'une industrie touristique autochtone forte. De surcroit, c'est toute l'industrie touristique au Quebec qui dépend de telles mesures.

C'est l'indéniable existence de l'industrie touristique autochtone au Quebec que, dans ce numéro de Téoros, nous avons voulu démontrer. Elle a le vent dans les voiles, malgré ses difficultés; et si le produit qu'elle offre n'est pas partout parfait, c'est que, justement, il devra sans cesse être peaufiné par ses promoteurs. Le potentiel, exceptionnel, ne peut se développer en vase clos : c'est dans l'harmonie entre son milieu d'émergence et l'ensemble de l'industrie que réside son avenir.

\section{Remerciements}

La coordination de ce numéro a été rendue possible grâce à la collaboration de la Société touristique Innu et, tout particulièrement, de sa directrice générale Guylaine Gill, que nous tenons à remercler. Pour la rédaction du présent numéro, nous avons beneficié des témoignages pertinents de gens $d$ 'affaires, de politiciens. de muséologues, d'animateurs et de leaders, tous autochtones, ainsi que de touristes qui ont bien voulu nous transmettre leurs commentaires. Chroniqueurs et analystes qui signent ces pages sont des gens qui auvrent depuis longtemps dans le domaine du tourisme et dont l'expérience s'assortit de solides connaissances du monde amérindien et inuit : nous souhaitons les remercier de leur précieuse contribution. Nous tenons également da rendre hommage aux pionniers de l'industrie touristique autochtone: Andrew Delisle chez les Mohawks, Aurelien Gill chez les Innu, Albert Diamond chez les Cris, la Fédération des coopératives du Nouveau-Québec chez les Inuit, Dominique Rankin chez les Algonquins, Max Gros-Louis chez les Wendat, ainsi que tous ceux qui, depuis plusieurs années, auvrent * dans l'ombre s à l'avènement d'une industrie touristique.

Míchel Noël, qui a coordonné la réalisation de ce numéro de Téoros, est un pionnier dans le développement du tourisme culturel en milieux autochtones. Il est ecrivain, ethologue, et, depuis de nombreuses années, Coordonnateur ministériel aux affaires autochtones au ministère de la Culture et des Communications, Bureau des sous-ministres, Gouvernement du Québec. 